(165)

\title{
Butterfly Assemblages in Two Different Habitats in Lowland Wet Zone, Sri Lanka
}

\author{
Peiris M.U.H. ${ }^{1 *}$, Pallewatta N. ${ }^{1}$, Dangalle C.D. ${ }^{1}$, Wijesundara S. ${ }^{2}$ \\ 'Department of Zoology \& Environment Sciences, University of Colombo, Sri Lanka, \\ ${ }^{2}$ National Institute of Fundamental Studies, Sri Lanka \\ *udarihpeiris@gmail.com
}

\begin{abstract}
Tropical butterflies should be studied as targets of conservation as they contribute towards maintenance of ecosystem functions including pollination. This study investigated butterfly assemblages of different habitats of the Western Province in Colombo district in Avissawella region. Seethawaka Wet Zone Botanical Garden (SWBG) lies adjacent to the Indikada Mukalana Forest Reserve (IMFR). In SWBG, a disturbed forest habitat (DFH) and a buffer zone habitat (BZH) which was located between the SWBG and the IMFR were selected as two different habitats. In both, 100m length fixed transects were established and all butterfly species within $10 \mathrm{~m}$ x $10 \mathrm{~m}$ x $100 \mathrm{~m}$ box were recorded from July to November 2016. Aerial nets and fruit baited traps were also used to sample butterflies. Shannon-Wiener diversity indices, evenness, species richness and relative abundance of butterflies were calculated for both habitats. A total of 62 butterfly species ( 6 endemic species and 15 endemic sub species) belonging to 6 families were recorded from the study. All six endemics are found in the DFH and only three were found in BZH. Species richness of two different habitats was equal (49 species) where higher abundance of butterflies (386 individuals) was in the DFH. BZH had 270 individuals. Shannon-Wiener diversity index and the evenness were highest in the BZH $(3.3109,0.8507)$ than the DFH $(2.7943,0.7179)$. Nymphalidae was the most abundant family throughout the study period whereas Family Riodinidae had the least number of individuals in both habitats. The data showed the presence of $25.1 \%$ of the butterflies of Sri Lanka and $32.1 \%$ of the lowland wet zone butterflies. Some belonged to Near Threatened and Vulnerable categories of conservation with the Ceylon Rose (Pachliopta jophon) being endangered and the Philippine Swift (Caltoris philippina) being Critically Endangered. Assemblages of butterflies recorded exhibit a range of feeding strategies (plant feeders, fruit feeders etc.) and depend on a wide range of host plants found in these habitats. The DFH is part of the highly visited SWBG. This study demonstrates the contribution made by a botanical garden originally established to conserve and display plants, to conservation of a major group of Sri Lankan invertebrates.
\end{abstract}

Keywords: Butterfly assemblages, Lowland wet zone, Endangered, Critically endangered, Sri Lanka

Proceedings of the $22^{\text {nd }}$ International Forestry and Environment Symposium 2017 of the Department of Forestry and Environmental Science, University of Sri Jayewardenepura, Sri Lanka 